\section{GREQAM}

Groupement de Recherche en Economie Quantitative d'Aix-Marseille - UMR-CNRS 6579

Ecole des Hautes Etudes en Sciences Sociales Universités d'Aix-Marseille II et III

\title{
ENDOGENOUS LINK STRENGTH IN DIRECTED COMMUNICATION NETWORKS
}

\section{Frédéric DEROÏAN}

September 2006

Document de Travail $n^{\circ}$ 2006-33 


\title{
Endogenous Link Strength in Directed Communication Networks
}

\author{
F. Deroian*†
}

September 4, 2006

\begin{abstract}
We present a model of communication network formation in which links' strengths are endogenously determined by individual incentives. Agents are endowed with a fixed amount of resource which they can distribute as they want in directed links. Individuals capture benefits from both direct and indirect access to others, in a way that takes into account the strength of each link. The wheel architecture is shown to be the unique efficient and stable architecture under mild restriction.
\end{abstract}

JEL Classification Numbers: D85, C70

Keywords: Directed Communication Network, Endogenous Link Strength, Efficiency, Stability

\section{Introduction}

The growth of communication good industries, such as the Internet, mobile phones and computers, and more generally all numeric goods, raises new economic issues

*The author is at GREQAM, Université d'Aix-Marseille, 2 rue de la Charité 13002 Marseille, France.

${ }^{\dagger} \mathrm{I}$ wish to thank F. Bloch for many valuable discussions and comments about this work. 
related to the formation of communication networks. At least two major queries are unavoidable: how do communication networks evolve through time? Do they maximize some collective outcome? One key aspect is that network formation is not supervised by any formal institution; rather, individuals decide whether to contribute to the shaping of such networks. This is therefore important to understand the individual incentives to join such networks. A second key aspect is that in many cases agents choose how much effort to devote to communicating in the network, and the level of investment in two distinct connections is not necessarily homogenous. For instance, as visiting your favorite web sites, you may decide how much time to spend on each, and in many circumstances you would not choose an homogenous distribution of time. That is, the link strength (as measured by the level of time, effort, money) is often endogenous to individual decisions. Third, many networks are directed. For instance, this may fit situations like visiting a web site, but consider also the (directed) relationship in a firm between a manager and a subordinate. With regard to non directed networks, a generic difference govern the formation of connections: in the latter case link formation is a bilateral action, while in the former case it is a unilateral action. Put differently, the coordination problem inherent to the non directed case vanishes in the directed case.

We address the three points in a simple model of network formation. This model complements Bloch and Dutta (2005)'s work on non directed network, so our setting is close to theirs. First, agents have a fixed and infinitely separable amount of resource that they can distribute over links. This may represent some effort, time or money spent in each relationship. Second, agents capture indirect benefits from accessing others, and this benefit depends on the level of investments made by intermediaries. We suppose that if there is a (directed) path linking agent $i$ to agent $j$, the value for agent $i$ stemming from that path is a function of the investments made by all intermediaries on that path; and we assume that the benefit she obtains from agent $j$ is the greatest value among all possible paths of finite length. In that context, we show that the wheel network is the unique efficient and stable (Nash) architecture. Our resource-constraint 
environment is therefore sufficient to generate a unique architecture making compatible private incentives and collective outcome. This remarkable coincidence between Nash and efficient networks is inherent to our resource-constraint environment. In our setting agents prefer to concentrate their allocation on the partners who provide them with the greatest indirect benefits. This results in the formation of the unique architecture such that agents access others through a chain of maximal length and composed of weights equal to amount $X$. We also present two basic extensions: we first propose a more general setting in which the results keep true, then we set up a simple dynamic process of network formation leading to the wheel architecture with probability 1.

This work can be related to studies devoted to the formation of communication networks ${ }^{1}$, or to enlarged context of directed network formation ${ }^{2}$. Among them, we discuss the closest articles.

Our contribution can be seen as an extension of Bloch and Dutta (2005) to directed networks. Indeed, the authors study the formation of a non directed communication network with endogenous link strength. In their setting, agents have a budget constraint and choose how much to invest in each relationship. Also, players obtain indirect benefit by the path maximizing the product of link strength (in the main part of the paper). The difference between the models is the following: in Bloch and Dutta's work, links are non directed and the value of a link depends on the level of investment exerted by each partner (investments are either substitutable or complementary). That is, link formation exhibits an intrinsic coordination problem which vanishes in our directed context. The authors show that substitutable investments promote the efficiency and stability of the star architecture, while complementary investments promote that of connected architectures (especially, the circle architecture is always stable and efficient for small populations). The reason why connected networks

\footnotetext{
${ }^{1}$ See for instance Jackson and Wolinsky (1996), Bala and Goyal (2000), Deroian (2003), Bloch and Dutta (2005), Kim and Wong (2006), Matsubayashi and Yamakawa (2006).

${ }^{2}$ See Dutta and Jackson (2000).
} 
emerge in that latter context is that in a tree, any two end-node agents have always an incentive to coordinate and invest part of their resource (the part exceeding the investment of their current partner) in the formation of a new link.

The second related paper, the discussion of which is presumably of first interest, is the one-way flow model of Bala and Goyal (2000). In this model agents form directed connections and obtain benefit from both direct and indirect access to other agents. But the resource scarcity inherent to our context induces a clear distinction: in the one-way flow model (without decay), any agent connected to a component receives a benefit written as a function of the number of agents in the component, whatever the structure of the component. This favors multiple individual best-responses and gives rise to multiple Nash architectures. In contrast, in our context the very structure in which agents are embedded matters and the structure of the game is sufficient to select a unique Nash architecture.

To finish, Kim and Wong (2006) propose a directed communication model in which agents get benefit from communicating with others. Two agents communicate with each other whenever there exists a cycle in which both are inserted. When agents are homogenous, the wheel architecture is uniquely determined by this solution concept. Our model is very distinctive in its foundations: first we do not integrate link formation costs, but rather agents invest a fixed amount of resource in links. Second, the endogenous link strength is specific to our context. Last, in our model agents capture benefit from accessing other agents, independently of whether or not others access them, i.e. value creation is not related to path circularity.

The article is organized as follows. Section 2 introduces the model. The next two sections respectively present the three-player case and the general case. Section 5 presents two extensions and section 6 concludes. All proofs are presented at the end of the note. 


\section{The model}

Let $N=\{1, \ldots, n\}$ be a set of agents. Every agent is endowed with a finite amount $X \in[0,1[$ of resources that can be devoted to establishing and maintaining links. Agents derive benefit from accessing other agents, either directly or indirectly. The key distinction with Bloch and Dutta (2005) is that the return of the investment made by agent $i$ on agent $j$ does not depend on how much agent $j$ has invested on agent $i$. Hence benefits are one-sided, in the sense that agents access others through valued directed paths. A valued directed network represents the structure of allocations.

Directed networks and individual strategies. A strategy of agent $i$ is a vector with $n-1$ coordinates $x_{i}=\left(x_{i, 1}, \ldots, x_{i, i-1}, x_{i, i+1}, \ldots, x_{i, n}\right)$ where $x_{i, j} \in[0, X]$ for each $j \in N \backslash\{i\}$, under the constraint that $\sum_{j \neq i} x_{i, j} \leq X$. Agent $i$ forms a link with $j$ if $x_{i, j}>0$. The set of strategies of agent $i$ is denoted by $X_{i}$. The set $X=X_{1} \times \ldots \times X_{n}$ is the space of strategies of all agents. A strategy profile $x=\left(x_{1}, \ldots, x_{n}\right)$ can be represented as a directed and valued network $g$. We denote by $G$ the set of (non negatively) weighted directed networks. The link $x_{i, j}>0$ is depicted as an edge between agents $i$ and $j$ with arrowhead pointing at agent $j$, and we denote by $i j$ this directed and valued link for convenience when there is no confusion; thus $i j \in g$ means that $x_{i, j}>0, i j \notin g$ means that $x_{i, j}=0$. A path between agent $k_{0}$ and agent $k_{p}$ is a sequence of links $k_{0} k_{1}, \cdots, k_{p-1} k_{p}$ without loops and such that $\prod_{q=0}^{q=p-1} x_{k_{q}, k_{q+1}}>0$. The distance from agent $i$ to agent $j$, and denoted $d(i, j ; g)$, represents the length of the shortest path from agent $i$ to agent $j$ in the network $g$. By convention if there is no finite path length the distance is infinite. We notice by $d_{\max }(i ; g)=\max _{j \neq i, d(i, j ; g)<+\infty} d(i, j ; g)$ the largest among all finite distances from agent $i$ to others in the network $g$.

Individual payoffs. In agreement with the literature on communication networks, we assume that the benefit of an agent in a network is given by the sum of the benefits she receives from accessing every other agent in her component. The benefit $b(i, j ; g)$ that agent $i$ captures from accessing agent $j$ is the maximal value of the product of 
link strength over the set $P(i, j ; g)$ of all finite paths from agent $i$ to agent $j$ :

$$
b(i, j ; g)=\max _{p \in P(i, j ; g)} x_{i a_{1}} \cdots x_{a_{m-1} j}
$$

with the convention that $b(i, j ; g)=0$ if there is no finite path from agent $i$ to agent $j$ in the network $g$. Note that the value of any path is less than 1. Agent $i$ 's payoff in the network $g$ is finally written $\pi_{i}(g)=\sum_{j \neq i} b(i, j ; g)$. In this formulation individual payoffs are additive with respect to the benefits obtained from each accessed agent.

Efficiency and stability. The welfare $W(g)$ of a network $g$ is the sum of individual payoffs on that network, i.e. $W(g)=\sum_{i \in N} \pi_{i}(g)$. A network is said efficient if there is no other network such that the sum of individual payoffs exceeds that of the former, i.e. $W(g) \geq W\left(g^{\prime}\right)$ for all $g^{\prime} \in G$. Further, we apply the usual Nash criterion: a network is Nash if for every agent, her current strategy is a best-response to the current strategies of all other agents. Formally, a profile of individual strategies $x^{*}=\left(x_{1}^{*}, \cdots, x_{n}^{*}\right)$ is a Nash equilibrium of the game if and only if, for every agent $i \in N$, for every strategy $x_{i} \in X_{i}, \pi_{i}\left(x_{i}^{*}, x_{-i}^{*}\right) \geq \pi_{i}\left(x_{i}, x_{-i}^{*}\right)$.

\section{The three-player case}

There are 16 architectures with three agents. Let us begin with efficiency issue. The welfare value of network architecture $g$ is the maximum welfare that can be obtained on that architecture over all possible allocations. An efficient network basically satisfies that every agent invests her total resource $X$ over her partners ('no waste' condition). The following notation will be useful: sometimes, the best allocation is such that certain agents reduce the weight of some link to zero; that is, the best alternative consists in modifying the architecture. If $z$ represents the value the architecture asymptotically tends to, we will notice $W(g) \rightarrow z$ (and not ' $=$ '). This means that the value is not reached by this architecture although its value tends to that. This precludes de facto this architecture from being efficient.

Figure 1 depicts the 16 architectures with three players and gives their values: The 


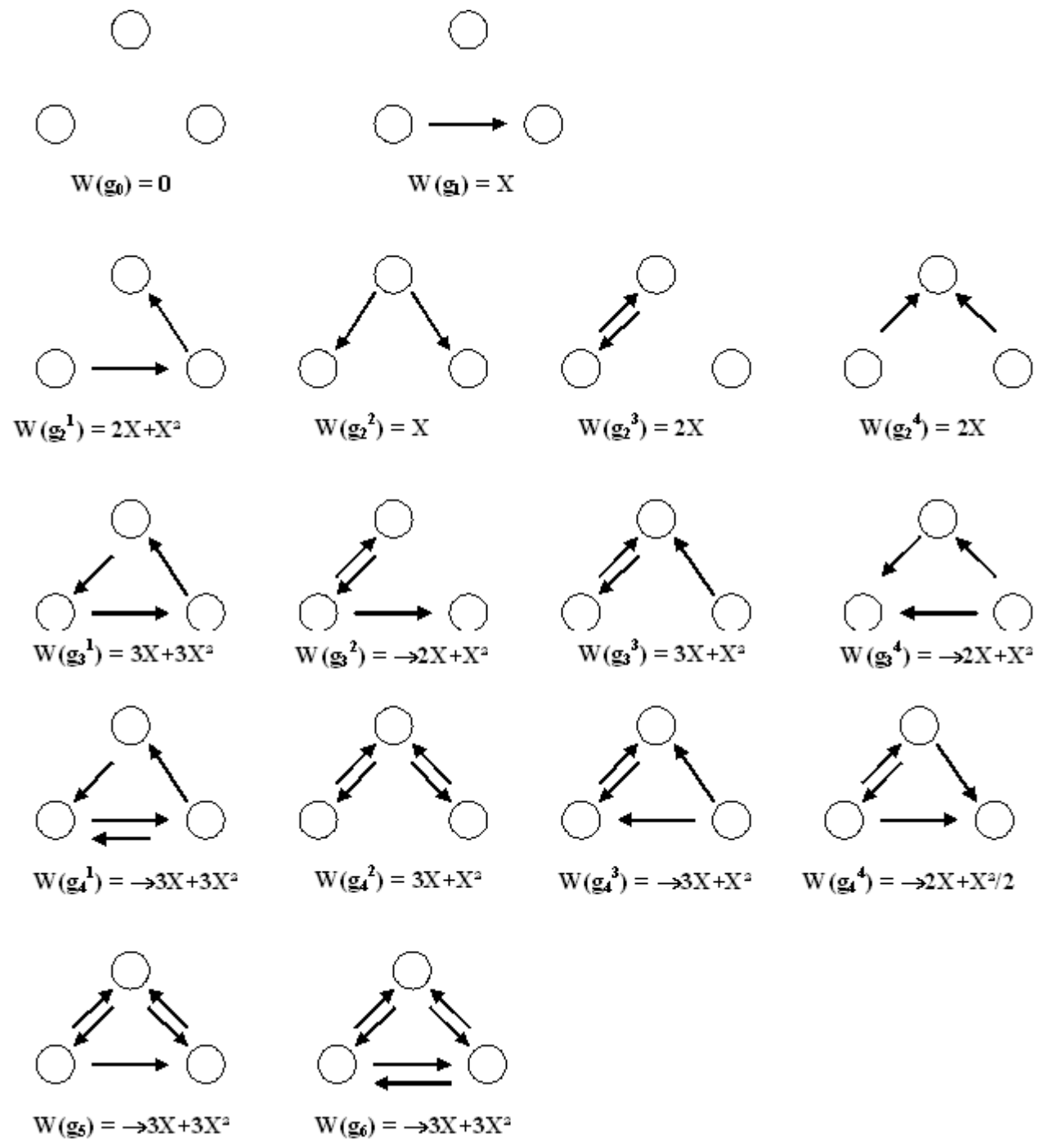

Figure 1: The 16 three-player architectures and their values

only efficient architecture is the wheel (labeled $g_{3}^{1}$ in the table), whose value is $W\left(g_{3}^{1}\right)=$ $3 X+3 X^{2}$.

Let us turn to stability analysis. Given that there is no link formation cost, a Nash network basically satisfies that each agent forms at least one link; then we restrict attention to networks containing at least 3 links. Furthermore, for each agent in a Nash network, the sum of all direct benefits equals $X$ (no waste). Hence, a Nash strategy maximizes the indirect benefits that the agent can capture. 
- 3-link architectures: the wheel $g_{3}^{1}$ (in which each link's weight is $X$ ) is Nash: indeed, for each agent the payoff is $X+X^{2}$, while any distribution of allocation $(x, X-x), x>0$ (the amount $x$ is invested on the link on which she currently invests $X$ ) would induce a payoff equal to either $X$ or $x(1+X)$ (depending on $X-x \lessgtr x X)$. The architectures $g_{3}^{2}$ and $g_{3}^{4}$ are not Nash, as one can identify one agent forming no link. Also $g_{3}^{3}$ is not Nash, as some agent (that with one inward connection) finds profitable to replace her current link with one entailing the formation of the wheel.

- 4-link architectures: none is Nash. In $g_{4}^{1}$ the agent with two links is better off by investing all on the link such that the deletion of the other link entails the formation of the wheel. In $g_{4}^{2}$, note that the two symmetric agents (forming one link) invest $X$ on their current connection, while the agent with two links shares her allocation into two parts. Therefore, the indirect benefit of a one-link agent is less than $X^{2}$. By replacing her link with a connection (of weight $X$ ) toward the third agent provides her with an indirect benefit equal to $X^{2}$. In $g_{4}^{3}$ the agent forming two links is better off by investing all in one link (benefiting therefore from indirect benefit). Last, in $g_{4}^{4}$ one agent does not form any link.

- the 5-link architecture: $g_{5}$ is not Nash; some agent (that forming two links and with one inward link) finds profitable to cut the link with the agent who do not form a link with her.

- the 6-link architecture: $g_{6}$ is not Nash; investing all on a single agent is strictly beneficial, since a positive benefit is in that case obtained from the agent at distance two.

To sum up, our environment exhibits no tension between stability and efficiency in the three-player case.

\section{The general case}

Our first result pertains with efficiency. We will use the following lemma: 
Lemma 1 For every agent $i$ in a network $g, \pi_{i}(g) \leq \sum_{k=1}^{d_{\max }(i ; g)} X^{k}$.

Considering any agent $i$ in some network $g$, lemma 1 guarantees that her payoff does not exceed that obtained by some agent starting a chain of length $d_{\text {max }}(i ; g)$ and composed of links with weights equal to $X$. The reason lies in the resource-constraint nature of our model. When the individual resource constraint is saturated, forming an additional link requires to decrease the investment put over some current links. This entails the following result: whatever a given set of paths of length $k$ issued from some agent $i$, the sum of their values cannot exceed that of one unique path of length $k$ and composed of maximal weights. The following proposition derives the set of efficient architectures:

Proposition 1 The complete wheel is the unique efficient network.

Our first conclusion is that a unique architecture is socially desirable. Indeed, this architecture is such that all agents access others by the means of a chain of length $n-1$ and composed of links of weight equal to $X$ (when all individual resource constraints are saturated). Since no finite distance can be larger than $n-1$, we use lemma 1 to conclude that no individual payoff can exceed that obtained in the wheel architecture. Uniqueness is derived by remarking that the wheel is the unique architecture such that all agents can obtain that maximal payoff (one can even see that whatever a distinct architecture, at most one agent can obtain such a payoff).

Our second result ensures that there is no tension between stable and efficient networks. Indeed,

Proposition 2 The complete wheel is the unique Nash network.

The proof relies on two points: First, we consider one agent with maximal payoff on the network, say agent $i$, and one agent at maximal distance from her, say agent $j$; noticing that agent $j$ is not critical for agent $i$, we derive that (i) agent $j$ 's payoff is not smaller than that of agent $i$, and (ii) for obtaining equality, agent $i$ 's payoff must be exactly equal to $\sum_{k=1}^{d_{\max }(i ; g)} X^{k}$ (we know from lemma 1 that it cannot be greater). We 
deduce that for each non terminal agent on the path from agent $i$ to agent $j$, she invests amount $X$ in a unique connection. Second, we notice that if some agent say $p$ is not accessed by agent $i$, then a possible strategy entails for agent $p$ a greater payoff than that of agent $i$, a contradiction. We deduce that agent $i$ observes all agents. Combined with the first point, we see that to join agent $j$, agent $i$ begins a chain of $n-1$ links with weight equal to $X$. We conclude that agent $j$ 's unique best response is to invest amount $X$ on agent $i$. The complete wheel is therefore uniquely selected.

Hence, a unique architecture is selected ${ }^{3}$ and there is a coincidence between efficient and stable networks. This result is in sharp contrast with the one-way flow model of Bala and Goyal (2000). The authors obtain in their benchmark case (without friction) that the complete wheel is both Nash and uniquely efficient, but many other architectures are also Nash (in their model Nash networks are minimally connected). Let us go back to our proof and see why the results of the two models diverge. In both models a Nash network is connected. In BG, in a connected network all agents observe all others, so they obtain the same gross benefit. That is, payoff differences only stem from distinct number of costly connections. This is not the case in our context, in which links are valued. This generates the following major distinction: consider some agent $i$ with maximal payoff and agent $j$ at maximal distance from agent $i$ (thus not critical to her). In both models, if agent $j$ forms a unique link with agent $i$, she obtains the same payoff as agent $i$ (hence, in both models at least two agents - i.e. agents $i$ and $j$ form a unique connection). But in BG, the fact that agent $i$ 's payoff is not smaller than that of agent $j$ does not impose the existence of a chain from agent $i$ to agent $j$ : connectedness is sufficient. This originates the multiplicity of Nash networks. In our resource-constraint setting, different connected architectures generate different

\footnotetext{
${ }^{3}$ The result on stability is basically robust to stability criteria allowing for collective deviations, like strong Nash equilibrium (Dutta and Mutuswami [1997]) or Strong stability (Jackson and van den Nouweland [2005]). Indeed, we know that (i) the wheel architecture ensures to all agents the maximal individual payoff and (ii) for any distinct architecture at most one agent obtains such a payoff.
} 
payoffs. The only compatible network must contain a chain of maximal length from agent $i$ to agent $j$ (and maximal weight $X$ on each arc), otherwise the presence of multiple branches lowers too much agent $i$ 's payoff, contradicting that her payoff is maximal.

\section{Extensions}

\subsection{A more general setting on the value of paths}

We define the set $\Psi_{l}(i, j ; g)$ of all paths of length $l$ from agent $i$ to agent $j$ in the network $g$ :

$$
\left.\left.\Psi_{l}(i, j ; g)=\left\{\left(x_{a_{0} a_{1}}, \cdots, x_{a_{l-1} a_{l}}\right) \in\right] 0, X\right]^{l} / a_{k} \neq a_{k^{\prime}} \forall k \neq k^{\prime}, a_{0}=i, a_{l}=j\right\}
$$

This formulation excludes paths containing some arcs of weight equal to zero. Then we define a function

$$
\left\{\begin{array}{l}
\phi_{l}: \Psi_{l}(i, j ; g) \longrightarrow \mathcal{R}_{+} \\
\left(x_{i a_{1}}, \cdots, x_{a_{l-1} j}\right) \longmapsto \phi_{l}\left(x_{i a_{1}}, \cdots, x_{a_{l-1} j}\right)
\end{array}\right.
$$

with $l$ arguments in $] 0, X]$, and generating a positive real number: $\phi\left(z_{1}, \cdots, z_{l}\right)>0$, i.e. a path (composed with positive weights) has positive value. Second, we suppose that the function is increasing and (weakly) convex in each argument. By convexity, we mean that for every indexes $l$ and $p$ :

$$
\phi_{l}\left(a_{1}, \cdots, a_{p}, \cdots, a_{l}\right)+\phi_{l}\left(a_{1}, \cdots, b_{p}, \cdots, a_{l}\right) \leq \phi_{l}\left(a_{1}, \cdots, a_{p}+b_{p}, \cdots, a_{l}\right) .
$$

The benefit obtained by agent $i$ from agent $j$ is written:

$$
b(i, j ; g)=\max _{l=1, \cdots, n-1} \max _{p \in \Psi_{l}(i, j ; g)} \phi_{l}(p)
$$

with the convention that $b(i, j ; g)=0$ whenever there is no finite path length from agent $i$ to agent $j$. For instance, the individual payoff in the complete wheel is written

$$
\sum_{k=1}^{n-1} \phi_{k}(\underbrace{X, \cdots, X}_{k \text { times }})
$$


Indeed, there is a unique finite path from agent $i$ to any agent $j$, and no two paths have equal length. Note that in this formulation we do not impose any ranking on the functions $\phi_{k}$, we only impose positivity.

Among all paths starting from agent $i$ and issuing to agent $j$, we denote with $P_{i j}^{*}(g)$ the subset of them with greatest value, i.e.

$$
P_{i j}^{*}(g)=\left\{p_{i j}(g) \in \operatorname{argmax}_{\Psi_{1}(i, j ; g), \cdots, \Psi_{n-1}(i, j ; g)} \phi_{l}(p)\right\} .
$$

For convenience, we arbitrarily select a unique best path, say $\Theta(i, j ; g) \in P_{i j}^{*}(g)$. We denote for each agent $i$ in the network $g$ the profile $P_{i}^{*}(\Theta, g)=\{\Theta(i, j ; g)\}_{j \neq i}$. This profile associates with each agent $j \neq i$ one path from $i$ to $j$ of greatest value. We define the set $\Gamma_{i}^{l}(\Theta, g)$ composed of the paths in $P_{i}^{*}(\Theta, g)$ of length $l$. Then, convexity implies the following lemma (this lemma is a direct extension of lemma 1):

Lemma 2 For every index $l \in\{1, \cdots, n-1\}$, and whatever selection process $\Theta$, we have:

$$
\sum_{p \in \Gamma_{i}^{l}(\Theta, g)} \phi_{l}(p) \leq \phi_{l}(\underbrace{X, \cdots, X}_{l \text { times }})
$$

This lemma bounds above the sum of benefits that agent $i$ captures from accessing agents placed at a given finite distance from her. To fix ideas, consider agent $i$ 's payoff in the following network (see figure 2):

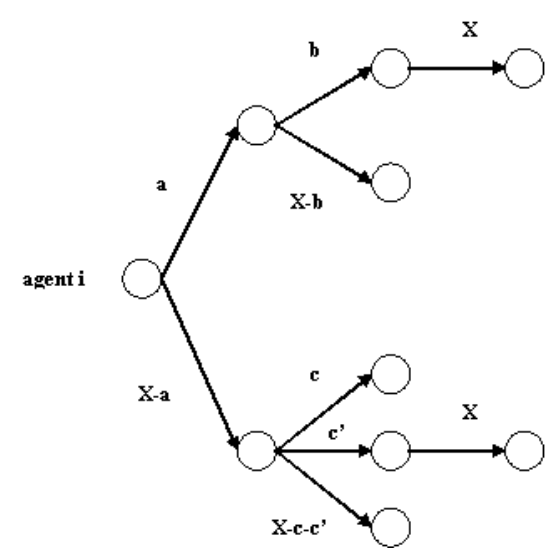

Figure 2: 
In this example $P_{i j}^{*}(g)$ is reduced to a unique element for any $j \neq i$. We obtain:

$$
\begin{gathered}
\pi_{i}=\phi_{1}(a)+\phi_{1}(X-a) \\
+\phi_{2}(a, b)+\phi_{2}(a, X-b)+\phi_{2}(X-a, c)+\phi_{2}\left(X-a, c^{\prime}\right)+\phi_{2}\left(X-a, X-c-c^{\prime}\right) \\
+\phi_{3}(a, b, X)+\phi_{3}(X-a, c, X)
\end{gathered}
$$

As the function is increasing,

$$
\left\{\begin{array}{l}
\phi_{3}(a, b, X) \leq \phi_{3}(a, X, X) \\
\phi_{3}(X-a, c, X) \leq \phi_{3}(X-a, X, X)
\end{array}\right.
$$

Further, the function is convex, so

$$
\left\{\begin{array}{l}
\phi_{1}(a)+\phi_{1}(X-a) \leq \phi_{1}(X) \\
\phi_{2}(a, b)+\phi_{2}(a, X-b) \leq \phi_{2}(a, X) \\
\phi_{2}(X-a, c)+\phi_{2}\left(X-a, c^{\prime}\right)+\phi_{2}\left(X-a, X-c-c^{\prime}\right) \leq \phi_{2}(X-a, X)
\end{array}\right.
$$

That is,

$$
\pi_{i} \leq \phi_{1}(X)+\phi_{2}(a, X)+\phi_{2}(X-a, X)+\phi_{3}(a, X, X)+\phi_{3}(X-a, X, X)
$$

Using again the convexity of $\phi_{2}$ and $\phi_{3}$, we obtain

$$
\pi_{i} \leq \phi_{1}(X)+\phi_{2}(X, X)+\phi_{3}(X, X, X)
$$

Hence, confirming lemma 3, we note that for each distance $l=1,2,3$, the sum of benefits that agents $i$ captures from agents placed at distance $l$ from her is bounded above by the value $\phi_{l}(X, \cdots, X)$.

The following lemma extends lemma 2:

Lemma 3 Whatever network $g \in G$ distinct from the complete wheel, there is at most one agent with payoff equal to $\sum_{k=1}^{n-1} \phi_{k}(X, \cdots, X)$. Hence at least $n-1$ agents have a payoff strictly smaller than that they obtain in the wheel.

As a direct consequence of lemmata 3 and 4 : 
Proposition 3 The complete wheel is both Nash and uniquely efficient.

Uniqueness (of efficiency set) comes from the fact that the complete wheel is the unique architecture providing $\phi_{k}(X, \cdots, X)$ from $k=1$ up to $k=n-1$ as benefit to all agents. Hence, we observe that a crucial determinant of uniqueness is that all functions $\phi_{k}(X, \cdots, X)$ are positive.

We obtain uniqueness of the stable set under the following more specific environment:

$$
\phi_{l}\left(x_{i a_{1}}, \cdots, x_{a_{l-1} j}\right)=\theta\left(x_{i a_{1}}\right) \times \cdots \times \theta\left(x_{a_{l-1} j}\right)
$$

with function $\theta():. \mathcal{R}_{+} \longrightarrow[0,1[$, increasing and convex, and such that $\theta(x)>0$ iff $x>0$. For instance, consider the function $\theta(x)=\delta \cdot x, \delta \in[0,1]$ and $x \in[0, X], X<1$. We obtain here a geometric decay function, in the sense that the value of a path $\left(x_{i a_{1}}, \cdots, x_{a_{l-1} j}\right)$ is $\delta^{l} \cdot x_{i a_{1}} \times \cdots \times x_{a_{l-1} j}$.

The additional result obtains:

Proposition 4 In such a specification, the complete wheel is the unique Nash network. Indeed, when $\phi$ is a product of identical functions, the proof of proposition 2 is easily extended.

\subsection{A dynamic process of network formation}

We present a dynamic process close to that presented in Bala and Goyal (2000) and pursued with slight modification by Kim and Wong (2006). We consider some initial network $g^{0} \in G$ and a discrete time sequence $1, \cdots, t, \cdots$ At each time $t$, a strict and non empty subset $A^{t}$ of agents is stochastically drawn from the population; the selected agents $i \in A$ play with probability 1 a weakly payoff-improving strategy; this includes possibility of playing the same strategy as the current one. The resulting network is labeled $g^{t}$. In our process, the new strategies are payoff-improving, but not necessarily best-responses (in BG they are such). In terms of individual informational requirements, our hypothesis is less demanding than that of BG (the authors 
have also developed in a working paper some analysis under alternative informational assumptions - see footnote 22 pp. 1192 in the related article).

The following result obtains:

Proposition 5 This dynamic process converges with probability 1 toward the wheel architecture, regardless the initial network it starts from.

The result is close to that of Bala and Goyal (2000) (or even Kim and Wong [2006]). Yet, our proof contains a difference inherent to our context, although similar in spirit. In $\mathrm{BG}$, the proof relies on the following mechanism: starting from any initial network, choose some agent $j_{1}$ with maximum profit on it, consider some agent $j_{2}$ furthest away from her (at maximal distance), and let agent $j_{2}$ form a unique connection with agent $j_{1}$. This strategy is a best-response: agent $j_{2}$ observes all the agents observed by agent $j_{1}$ while forming a unique connection. Then replicate the process by replacing agent $j_{1}$ by agent $j_{2}$ and by finding some agent at maximal distance from agent $j_{2}$. The process goes on until achievement of the wheel. In our context, starting from any network, choose some agent $j_{1}$ with maximum profit on it, consider some agent $j_{2}$ furthest away from her, and make agent $j_{2}$ invest all resource $X$ on agent $j_{1}$. Then she obtains at least agent $j_{1}$ 's payoff, and consequently she weakly improves her current payoff; now because links are valued, this new strategy may not be a best-response: indeed, she obtains exactly $X+X\left(\pi_{j_{1}}(g)-b\left(j_{1}, j_{2} ; g\right)\right)$ from the new strategy. It is easy to find a network containing another agent $j_{1}^{\prime}$ with maximal payoff, and such that both agent $j_{2}$ is furthest away from her and $b\left(j_{1}, j_{2} ; g\right)>b\left(j_{1}^{\prime}, j_{2} ; g\right)$.

\section{Concluding remarks}

We study a non-cooperative model of network formation in which agents invest a fixed and infinitely separable amount of resource into links. That is, the strength of links is endogenously determined by individual investment decisions. In this resourceconstraint setting, we find that the wheel architecture is both uniquely efficient and 
uniquely Nash. Therefore, this environment is sufficient to generate a unique architecture making compatible private incentives and collective outcome.

Further advances may investigate some lines of research: introducing convex cost of link formation generates an immediate trade-off between distance and cost. Relaxing homogeneity in agents' types may also constitute an interesting extension, as well as introducing information asymmetry.

\section{References.}

Bala, V., and S. Goyal, 2000, A Noncooperative Model of Network Formation, Econometrica, 68, 1181-1229.

Bloch, F. and B. Dutta, 2005, Communication networks with Endogenous Link Strength, mimeo GREQAM.

Deroian, F., 2003, Farsighted strategies in the formation of a communication network, Economics Letters, 80, 343-349.

Dutta, B. and M. O. Jackson, 2000, The Stability and Efficiency of Directed Communication Networks, Review of Economic Design, 5, 251-272.

Dutta, B. and S. Mutuswami, 1997, Stable networks, Journal of Economic Theory, 67, 322-344.

Jackson, M. and A. van den Nouweland, 2005, Strongly stable networks, Games and Economic Behavior, 51, 420-444.

Jackson, M. and A. Wolinsky, 1996, A Strategic Model of Economic and Social Networks, Journal of Economic Theory, 71, 44-74.

Kim, C. and K.-C. Wong, 2006, Network Formation and Stable Equilibrium, forthcoming in the Journal of Economic Theory.

Matsubayashi, N. and S. Yamakawa, 2006, A note on network formation with decay, forthcoming in Economics Letters.

\section{Proofs.}


Proof of lemma 1. The proof is given by induction.

Inductive argument $P(k), k \in\{1, \cdots, n-1\}$ : if $d_{\text {max }}(i ; g)=k$, then $\pi_{i}(g) \leq \sum_{q=1}^{k} X^{q}$. We trivially have $P(1)$. Suppose now $P(1), \cdots, P(k)$. We will derive $P(k+1)$. Indeed, if $d_{\max }(i ; g)=k+1$,

$$
\pi_{i}(g)=\sum_{j \neq i} x_{i j}(g)+\sum_{j \neq i} x_{i j}(g) \pi_{j}^{i}(g)
$$

where $\pi_{j}^{i}(g)$ represent the relevant part of agent $j$ 's payoff for agent $i$. That is, that derived from agents distant until at most order $k$ of agent $j$; if some agent $p$ is at distance $k+1$ or more than agent $j$, then either the distance is infinite and it does not count for agent $i$ 's payoff, or the distance is finite which means by construction that agent $p$ is at smaller distance from agent $i$ (since $\left.d_{\max }(i ; g)=k+1\right)$.

Since the relevant network used by agent $i$ to access others through agent $j$ is a tree starting from agent $j$ and with path length bounded above by $k$, we deduce as applying $P(1), \cdots, P(k)$ to agent $j$ (more precisely to the relevant part of agent $j$ 's payoff for agent $i$ ) that $\pi_{j}^{i}(g) \leq \sum_{q=1}^{k} X^{q}$. Hence,

$$
\pi_{i}(g) \leq \sum_{j \neq i} x_{i j}(g)+\sum_{j \neq i} x_{i j}(g) \sum_{q=1}^{k} X^{q}
$$

And since $\sum_{j \neq i} x_{i j}(g) \leq X$, we obtain that

$$
\pi_{i}(g) \leq \sum_{q=1}^{k+1} X^{q}
$$

which proves $P(k+1)$.

Proof of proposition 1 . We notice that a link $i j$ is not necessarily active in the sense that the link is used to extract benefit. For instance, consider the following three-player network such that $x_{12}=X-\epsilon, x_{13}=\epsilon, x_{23}=X$. Then agent 1 may join agent 3 with two paths. And the two-arc path worths more than the one-arc path whenever $(X-\epsilon) X>\epsilon$. This is true for sufficiently small values of $\epsilon$. But in such a case a more efficient network exists in which agent $i$ reallocates the investment $x_{i j}$ into the connection $x_{i k_{1}}$ where $i k_{1}$ is the arc used by agent $i$ to join agent $j$ (each agent 
using the path $i k_{1}, \ldots, k_{p} j$ to extract value would be better off, and no agent was using the link $i j$ to extract value by definition). Hence we can restrict attention to networks such that for every agent, the sum of benefits associated with agents at distance 1 from her is exactly equal to $X$.

From lemma 1 we easily derive that the complete wheel is efficient: in the wheel architecture all agents access others by the means of a chain of length $n-1$ and composed of links of weight equal to $X$ (when all individual resource constraints are saturated). Since no finite distance can be larger than $n-1$, we use lemma 1 to conclude that no individual payoff can exceed that obtained in the wheel architecture. We now derive uniqueness by showing that whatever network $g \in G$ distinct from the complete wheel, there is at most one agent with payoff equal to $X+\cdots+X^{n-1}$. Consider a network $g$ distinct from the complete wheel. Suppose without loss that $\pi_{1}(g)=X+\cdots+X^{n-1}$. Then there is a chain, starting from agent 1 and containing all agents, and all agents except the terminal node, say agent $n$, invest $X$ on her link. Thus, these agents have a unique connection. EITHER agent $n$ forms a set of links distinct from investing her total resource on agent 1 ; and clearly agents 2 to $n$ obtain less than agent 1's payoff; OR agent $n$ invests amount $X$ on agent 1 , in which case the complete wheel forms. We deduce that the complete wheel is the unique architecture such that all agents obtain a payoff equal to $X+X^{2}+\ldots+X^{n-1}$. We conclude that the complete wheel is uniquely efficient.

Proof of proposition 2. We notice that the wheel architecture with all weights equal to $X$ is Nash, since no other network can guarantee larger individual payoffs. We then turn to uniqueness. We first show that some agent with maximal payoff begins a chain composed of links of weight equal to $X$. Second we deduce that this agent accesses the whole society. Third we conclude that the wheel is uniquely selected.

Assume without loss that agent $i \in \operatorname{argmax}_{k \in N} \pi_{k}(g)$. Consider agent $j$ such that $d(i, j ; g)=d_{\max }(i ; g)$. Then agent $j$ is non critical to agent $i$. Hence, if forming a 
unique link toward agent $i$, agent $j$ obtains

$$
\pi_{j}=X+X\left(\pi_{i}(g)-b(i, j ; g)\right)
$$

since agent $i$ 's payoff is not affected by agent $j$ 's strategy. Recalling that agent $i$ 's payoff is maximal, we know that $\pi_{j}(g) \leq \pi_{i}(g)$, inducing $X+X\left(\pi_{i}(g)-b(i, j ; g)\right) \leq \pi_{i}(g)$. But $b(i, j ; g) \leq X^{d_{\max }(i ; g)}$. Hence the latter inequality implies that:

$$
\pi_{i}(g) \geq \frac{X}{1-X}\left(1-X^{d_{\max }(i ; g)}\right)
$$

or equivalently $\pi_{i}(g) \geq X+X^{2}+\ldots+X^{d_{\max }(i ; g)}$. But by lemma 1 agent $i$ 's payoff cannot exceed that value. Thus it must be the case that $\pi_{i}(g)=X+X^{2}+\ldots+X^{d_{\max }(i ; g)}$. We also derive two points. First $\pi_{j}(g)=\pi_{i}(g)$. Second, for agent $i$ 's payoff to be such, agent $i$ accesses all others through a chain of length $d_{\max }(i ; g)$ and composed of links with weights equal to $X$.

Suppose that some agent $p$ is not accessed by agent $i$, i.e. $d_{\max }(i ; g)<n-1$. Then by investing amount $X$ on agent $i$, agent $p$ obtains a payoff equal to $X+X \pi_{i}(g)$. As agent $i$ 's payoff is maximal, it must be the case that $X+X \pi_{i}(g) \leq \pi_{i}(g)$, which means that $\pi_{i}(g) \geq \frac{X}{1-X}$. But as $X<1, X+X^{2}+\ldots+X^{n-1} \leq \frac{X}{1-X}$, a contradiction. Hence, $d_{\max }(i ; g)=n-1$.

We know that there is a unique agent $j$ such that $d(i, j ; g)=n-1$ and that $\pi_{j}(g)=$ $\pi_{i}(g)$. Given that agent $i$ begins a chain of length $n-1$ and composed of links of weights equal to $X$, the unique strategy for agent $j$ to obtain agent $i$ 's payoff consists in forming a unique connection of weight $X$ with agent $i$. The complete wheel obtains.

Proof of proposition 5. The proof is given by proposing from any network $g^{0}$ a sequence of individual beneficial deviations at each period, and leading to the wheel network in a finite delay. We do not impose individual deviations to be best-response strategies; rather we demand that the new strategy does not deteriorate the individual payoff. 
Consider some initial network $g^{0} \in G$ and some agent $j_{1} \in \operatorname{argmax}_{k \in N} \pi_{k}\left(g^{0}\right)$, one agent with maximum profit on the network $g^{0}$. Choose some agent $j_{2}$ such that $d\left(j_{1}, j_{2} ; g^{0}\right)=d_{\max }\left(j_{1} ; g^{0}\right)$, so agent $j_{2}$ is furthest away from agent $j_{1}$ in the network $g^{0}$. This may include the possibility that $d\left(j_{1}, j_{2} ; g^{0}\right)=+\infty$. Clearly, agent $j_{2}$ is not critical to agent $j_{1}$ (dropping all links formed by agent $j_{2}$ does not affect agent $j_{1}$ 's payoff). Then by investing $X$ on agent $j_{1}$ in period $t=1$, that is if agent $j_{2}$ 's new strategy is $x_{j_{2}}^{\prime}=\left(0, \cdots, 0, x_{j_{2}, j_{1}}=X, 0, \cdots, 0\right)$ and $g^{1}=x_{j_{2}}^{\prime} \oplus g_{-o u t: j_{2}}^{0}$ the resulting new network $\left(g_{-o u t: j_{2}}^{0}\right.$ represents the network $g^{0}$ minus all links formed by agent $\left.j_{2}\right)$, agent $j_{2}$ gets a payoff $\pi_{j_{2}}\left(g^{1}\right) \geq \pi_{j_{1}}\left(g^{0}\right)$, so that the new strategy weakly improves agent $j_{2}$ 's payoff (i.e., $\pi_{j_{2}}\left(g^{1}\right) \geq \pi_{j_{2}}\left(g^{0}\right)$ ) (it strictly improves her payoff in the case $d\left(j_{1}, j_{2} ; g^{0}\right)=+\infty$ ). Consider now the network $g^{1}$. By construction, agent $j_{2} \in \operatorname{argmax}_{k \in N} \pi_{k}\left(g^{1}\right)$. Again, consider some agent $j_{3}$ such that $d\left(j_{2}, j_{3} ; g^{1}\right)=d_{\max }\left(j_{2} ; g^{1}\right)$; equivalently agent $j_{3}$ is such that $d\left(j_{1}, j_{3} ; g_{-i n: j_{2}}^{0}\right)=d_{\max }\left(j_{1} ; g_{-i n: j_{2}}^{0}\right)$, if $g_{-i n: j_{2}}^{0}$ denotes the network $g^{0}$ minus all links pointing at agent $j_{2}$ (again, it can be the case that $d\left(j_{1}, j_{3} ; g_{-i n: j_{2}}^{0}\right)=+\infty$ ). By the same argument, assume that agent $j_{3}$ is selected at time $t=2$ and that she invests all $X$ on agent $j_{2}$. Then she obtains at least the same profit, so her new profit is not less than the maximum profit in $g^{1}$, so this strategy is payoff-improving.

Computing the process further, we go through until selecting agent $j_{1}$ in a finite time, who similarly improves her payoff by completing the wheel architecture (no individual payoff exceeds that obtained in the wheel).

The proof is completed by remarking that the wheel architecture is the unique absorbing state of the process: first whatever network distinct from the wheel, at least one agent can improve her strategy (since the wheel network is uniquely Nash). Second, the wheel architecture is Nash strict; hence no agent in the wheel possesses another strategy giving her an equal payoff. 